\title{
Muscle strength and endurance to predict successful extubation in mechanically ventilated patients: A pilot study evaluating the utility of upper-limb muscle strength and ergometry
}

\author{
C R de Beer, ${ }^{1}$ BPhysT, MPhysT; A J van Rooijen, ${ }^{1}$ BSc (Fis), MSc, TOD (Fis), PhD Physiotherapy; J P Pretorius, ${ }^{2}$ MB ChB, MMed (Surg), \\ FCS (SA) Critical Care; P J Becker, ${ }^{3}$ MSc, PhD; P Rheeder, ${ }^{4}$ MB ChB, MMed, FCP (SA), MSc (Clin Epi), PhD; \\ F Paruk, ${ }^{2}$ MB ChB, FCOG (SA), Cert Crit Care (SA), PhD \\ ${ }^{1}$ Department of Physiotherapy, Steve Biko Academic Hospital and Faculty of Health Sciences, University of Pretoria, South Africa \\ ${ }^{2}$ Department of Critical Care, Steve Biko Academic Hospital and Faculty of Health Sciences, University of Pretoria, South Africa \\ ${ }^{3}$ Research Office, Faculty of Health Sciences, University of Pretoria, South Africa \\ ${ }^{4}$ Department of Internal Medicine, Steve Biko Academic Hospital and Faculty of Health Sciences, University of Pretoria, South Africa
}

Corresponding author: C R de Beer (crdebeer@gmail.com)

\begin{abstract}
Background. Successful extubation of mechanically ventilated patients is essential for the physiotherapist to succeed in respiratory rehabilitation of the patient. Delay in the weaning process increases the complication rate of mechanical ventilation. A variety of parameters are used as predictors of extubation readiness, but the association between muscle strength (deltoid, neck flexor muscle group and trapezius), endurance and extubation readiness has not been determined.

Objectives. The aim of the study was to determine if muscle strength and endurance can be used as possible predictors of successful extubation in mechanically ventilated patients. The objectives were to determine if muscle strength measured with the Oxford grading scale can be used as a possible predictor, and if muscle endurance measured with the MOTOmed letto2 cycle ergometer can be used as a possible predictor.

Methods. During the pilot study, 37 subjects were recruited. Deltoid, neck flexors and trapezius muscle strength was tested using the Oxford grading scale and respiratory muscle strength using maximum inspiratory and expiratory pressures. Endurance was determined by riding the MOTOmed letto2 cycle ergometer for 5 minutes with the upper limbs.

Results. Muscle strength of the deltoid and the neck flexor muscle group tested with the Oxford grading scale was associated with successful extubation respectively, $(p=0.022 ; p=0.019)$. Muscle endurance tested with the MOTOmed letto2 cycle ergometer also demonstrated an association with successful extubation $(p=0.014)$.

Conclusion. Future studies with larger sample sizes are recommended.

Keywords. Mechanical ventilation, intensive care unit, extubation failure, respiratory muscle strength, peripheral muscle strength, muscle endurance

S Afr J Crit Care 2018;34(2):44-49. DOI:10.7196/SAJCC.2018.v34i2.360
\end{abstract}

\begin{abstract}
Mechanical ventilators have revolutionised critical care in patients with respiratory failure, cardiac failure or requiring postoperative respiratory support. ${ }^{[1,2]}$ Failure to wean from mechanical ventilation within 48 hours is a significant clinical and economic problem; it has been associated with increased risk of developing nosocomial infections, airway trauma, respiratory muscle weakness, mortality and decreased endurance. ${ }^{[1,3,4]}$ It may also reduce the patient's functional ability and quality of life. ${ }^{[1,2,5]}$

Weaning from mechanical ventilation may comprise up to $40 \%$ of the time that the patient spends in an intensive care unit (ICU) ${ }^{[1,2,6]}$ Spontaneous breathing trials (SBTs) are used to judge the patient's readiness for extubation. ${ }^{[7]}$ Patients who are deemed capable of maintaining their airways are therefore usually extubated after completing the SBT successfully. Ten to $20 \%$ of patients who pass an SBT still fail extubation. ${ }^{[7-9]}$ Failed extubation has been defined as the need for re-intubation within 48 - 72 hours post extubation; ${ }^{[7]}$ this is associated with longer ICU stays, longer hospital stays, greater costs and higher mortality rates. ${ }^{[1,5]}$ The mortality rate in such situations ranges from $25 \%$ to $50 \% \cdot{ }^{[1,5,7]}$
\end{abstract}

De Jonghe et al. ${ }^{[10]}$ reported that a low maximum inspiratory pressure (MIP), maximum expiratory pressure (MEP) and Medical Research Council score (MRC-score) were independent predictors of delayed extubation. Inspiratory muscle training that improves respiratory muscle strength has been demonstrated to improve the weaning outcome. ${ }^{[3,4,1]}$ Given the combined nerve innervations of the respiratory muscles and the deltoid, neck flexors and trapezius muscles via the corticospinal pathway $(\mathrm{C} 1-\mathrm{C} 6),{ }^{[12,13]}$ the question arises whether upperlimb muscle strength and endurance could predict successful extubation in mechanically ventilated patients by means of interneuron activation. The present pilot study was conducted to determine if upper-limb muscle strength and endurance are potential predictors of successful extubation (primary outcome) and passing an SBT (secondary outcome).

\section{Objectives}

The objectives of this pilot study were (i) to determine if selected upper-limb muscle strength, measured with the Oxford grading 
scale, is a potential predictor of successful extubation; and (ii) to determine if muscle endurance, measured with the MOTOmed letto2 cycle ergometer, is a potential predictor of successful extubation.

\section{Methods \\ Study design and population}

An observational, cross-sectional pilot study was conducted from September 2015 to December 2015. Subjects were recruited from the surgery, trauma and medical ICUs at Steve Biko Academic Hospital, Pretoria, South Africa. The Research Ethics Committee, Faculty of Health Sciences, University of Pretoria, approved the protocol (number 309/2015). Subjects who were considered for the first time to be eligible for an SBT by the healthcare team according to the unit protocols, were evaluated to determine if they complied with the inclusion criteria. Inclusion criteria constituted: patients $\geq 18$ years old, awake and co-operative, mechanically ventilated for $\geq 3$ days, understand Afrikaans or English, haemodynamic stability including a heart rate (HR) $\leq 140$ beats per minute (BPM), systolic blood pressure $\geq 90 \mathrm{mmHg}$, haemoglobin $\geq 7$ $\mathrm{g} / \mathrm{dL}^{-1}$ and temperature $<38.5^{\circ} \mathrm{C}$. Minimal mechanical ventilator settings of continuous positive pressure ventilation, positive endexpiratory pressure (PEEP) $\leq 8 \mathrm{cmH}_{2} \mathrm{O}, \mathrm{FiO}_{2}$ $\leq 40 \%, \mathrm{SpO}_{2} \geq 90 \%$, no significant acidosis ( $\mathrm{pH}$ $\geq 7.35$ ) and $\mathrm{PaO}_{2} / \mathrm{FiO}_{2}>150$ were required. In addition, a good cough reflex to tracheal suctioning, a positive cuff test $(>110 \mathrm{ml}$ cuffleak volume) and no or minimal-to-moderate secretions ${ }^{[14]}$ (patients in the unit were standardly suctioned 4-hourly) were required. The exclusion criteria were: upper airway obstruction, body mass index $>35 \mathrm{~kg} / \mathrm{m}^{2},{ }^{[15]}$ cardiomyopathy, atrial fibrillation, primary/ previously diagnosed neuromuscular disorder, inability to co-operate, severe agitation (Richmond Agitation Sedation Scale (RASS) $\geq+2$ ) or an acute asthma attack. Subjects with spinal cord injuries, amputations, fractures, soft-tissue injuries, burns or dressings limiting the testing of muscle strength were excluded owing to physical assessment methods, e.g. Oxford grading scale. As standard practice, patients received daily physiotherapy treatment, including chest physiotherapy and rehabilitation. All participants gave voluntary informed consent.

\section{Study procedures}

Subjects were only evaluated once during the study. Recruitment is demonstrated in Fig. 1.

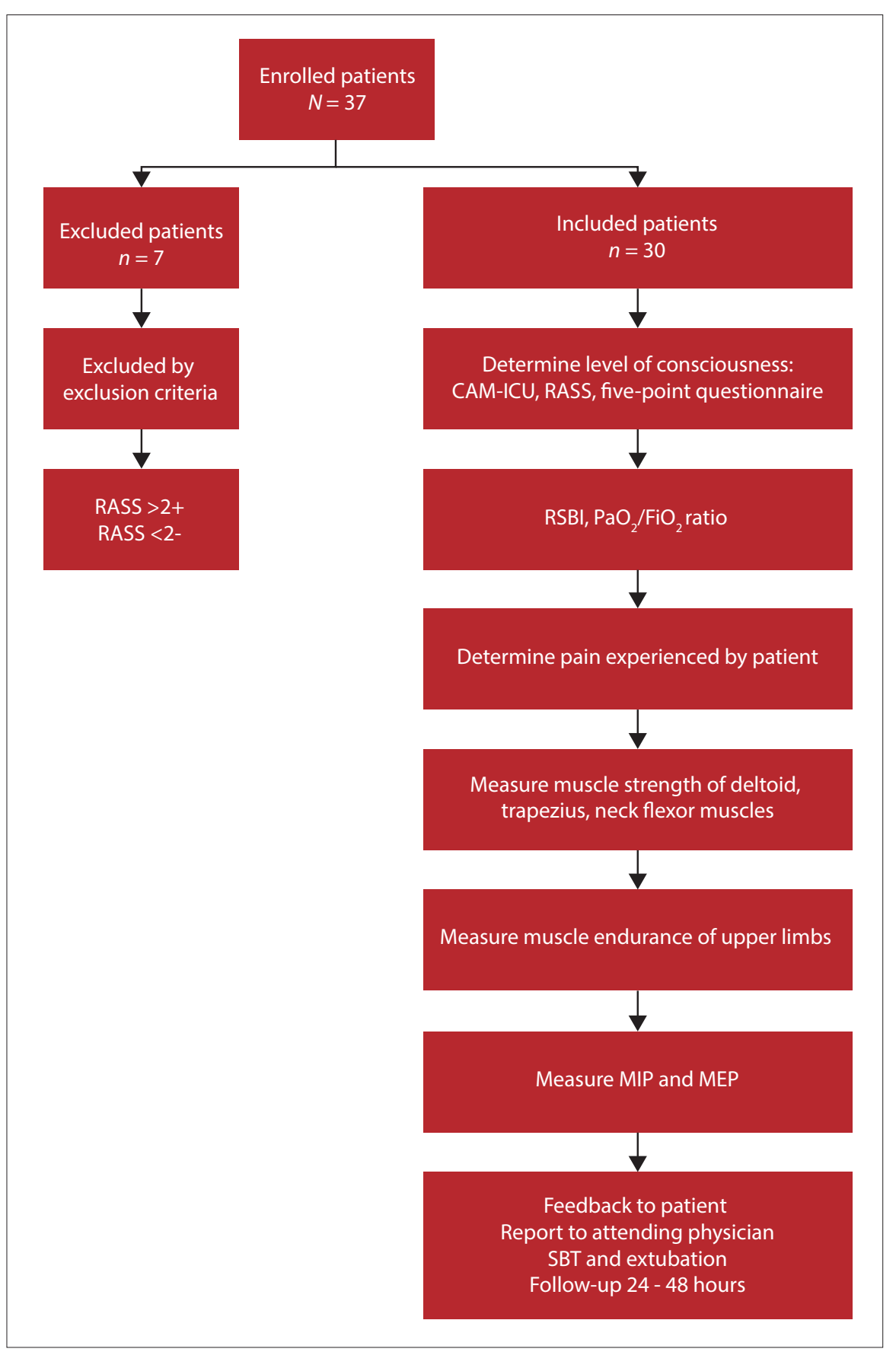

Fig. 1. Procedure from selection to participation. (CAM-ICU $=$ Confusion Assessment Method for intensive care unit; RASS = Richmond Agitation Sedation Scale; RSBI = rapid shallow breathing index; $M I P=$ maximum inspiratory pressure; $M E P=$ maximum expiratory pressure; $S B T=$ spontaneous breathing trial.)

Subjects considered ready for an SBT were evaluated with the Confusion Assessment Method for ICU (CAM-ICU) to determine their delirium status and cognitive ability. ${ }^{[16]}$ For the CAM-ICU to be negative, the RASS component needs to be 0 . Subjects with a RASS of $-1,0$ or +1 and an ability to answer 3 of the 5 questions in the 5 -point questionnaire ${ }^{[17]}$ were considered to be awake and co-operative by the team, a pre-requisite for manual muscle testing. ${ }^{[18]}$ The principal researcher measured peripheral muscle strength and endurance.
Two critical care clinical technologists recorded MIP and MEP values.

The Numeric Rating Scale (NRS) was used to evaluate the subjective experience of pain prior to muscle strength and endurance testing, with appropriate pain management ${ }^{[17]}$ implemented by nursing staff. The rapid shallow breathing index (RSBI) and $\mathrm{PaO}_{2} /$ $\mathrm{FiO}_{2}$ ratio were documented prior to muscle strength and endurance testing. Deltoid (middle fibres), neck flexors and trapezius (upper fibres) muscle strength was measured 
using the Oxford grading scale. ${ }^{[19]}$ The necessary movement was demonstrated before testing. Muscle strength testing was immediately discontinued if the subject showed any sign of haemodynamic instability ( $\mathrm{HR}>140 \mathrm{BPM}$, systolic $\mathrm{BP}<90 \mathrm{mmHg}, \mathrm{SpO}_{2}<90 \%$ ). Immediately after the muscle strength test, endurance was measured using the MOTOmed letto2 cycle ergometer (Fig. 2). Subjects rode the bicycle using their upper limbs while in the semi-Fowler's ( $45^{\circ}$ flexion) position in bed. The Servo cycle programme measured the distance and time rode actively (subject pushing the pedals) and passively (machine moving the pedals). The resistance was set at one gear (gear one is equal to $0.85 \mathrm{~kg}$ ) and the subjects rode the bicycle for 5 minutes. Exercise at one gear was considered suitable for both weaker and stronger subjects, as stronger subjects were able to increase their speed and active distance while riding the ergometer. In general, endurance testing for healthy persons can be used to determine an individual's ability to sustain highintensity exercise for longer than 4 minutes. ${ }^{[20,21]}$ Due to the subjects being critically ill, 5 is the minimum number of minutes needed to evaluate endurance. ${ }^{[20,21]}$ Endurance testing was stopped immediately if the subject exhibited any sign of haemodynamic instability. Subjects only rode the MOTOmed letto2 cycle ergometer once.

Available ventilators (Avea and Vela from Respiratory Care Africa) were used to record the best of 3 MIP and MEP readings immediately after riding the MOTOmed letto2 cycle ergometer. Subjects were subjected to an SBT 30 minutes after completion of muscle strength and endurance testing. Muscle strength and endurance testing results were not utilised to influence the decision to extubate the subject. Subjects who passed the SBT successfully according to the unit protocol (RR $<35$ BPM, HR <145 BPM, no major arrhythmias requiring venous drug therapy, systolic BP $<180 \mathrm{mmHg}$, no anxiety or agitation) were then extubated. Subjects were followed-up for $24-48$ hours after the SBT and/or extubation to determine if the SBT and/or extubation was successful. Subjects who failed the SBT were only evaluated once.

\section{Statistical analysis}

Association of primary outcome (extubation failure) and SBT (secondary outcome) with muscle strength was assessed using Fisher's exact test and

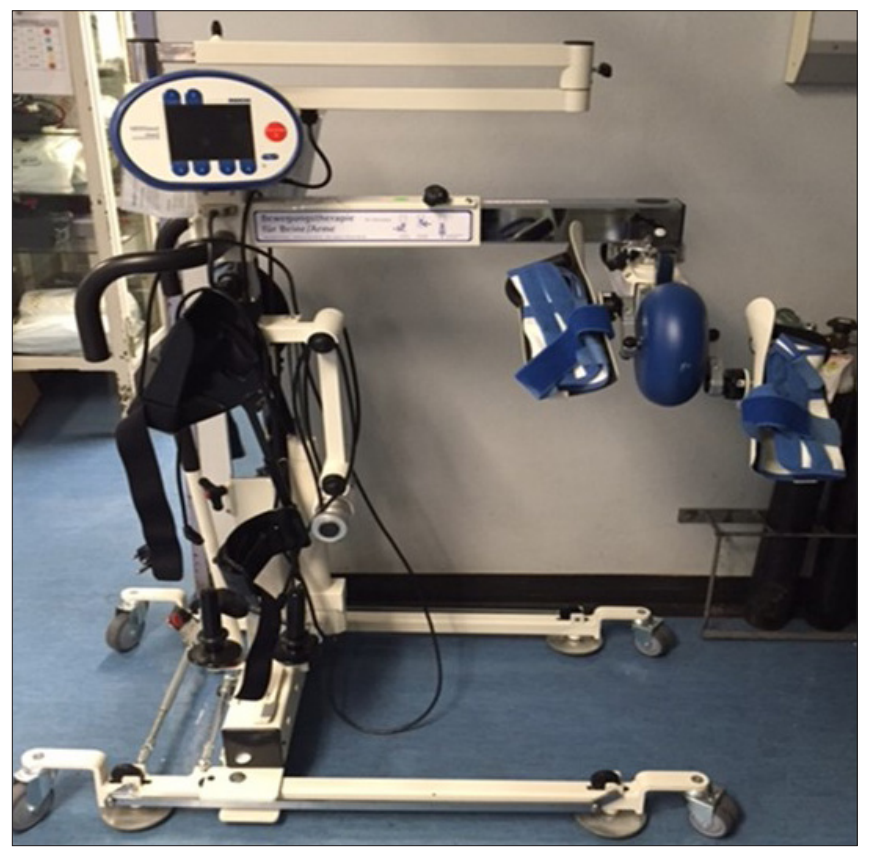

Fig. 2. MOTOmed letto 2 cycle ergometer. frequencies with percentages were reported by strength category over outcome. With respect to continuous parameters, outcome groups were compared using Wilcoxon's rank sum test and median was reported over outcome along with interquartile range (IQR). The agreement of SBT and extubation failure was assessed using McNemar's test for symmetry (test for bias) and Cohen's Kappa statistic for intra-rater agreement. Data were analysed using STATA version 15 software ${ }^{[22]}$ and R3.1.1. ${ }^{[23]}$ Data were expressed as mean, median, SD and (25th - 75th) percentiles. Results were significant if $p$-values $\leq 0.05$.

\section{Results}

Results are discussed according to the aim and objectives of the study. Thirty-seven potential subjects complied with the inclusion criteria. Seven subjects were, however, excluded after level of co-operation and cognition testing. RASS scores were either RASS $\geq+2$ or RASS $\leq-2$ and the subjects were not able to participate in muscle strength and endurance testing. There were 23 successfully extubated patients (11 male and 12 female) who were on average 45 years old. Two patients passed the SBT but failed extubation, and 5 failed the SBT and were not extubated. For the purposes of this study, the 7 patients $(1$ male and 6 female) who failed either the SBT or the extubation were grouped together for descriptive purposes (Table 1). The mean age of this group was 61 years; 5 were $\geq 59$ years old, and the remaining 2 patients who failed were 30 years old. The subjects' demographics and diagnoses are presented in Table 1.

Muscle strength testing of the deltoid and neck flexor muscles bilaterally with $p$-values $<0.05$ showed associations with extubation outcome (Table 2). There was no association between extubation outcome and bilateral upper trapezius muscle strength.

Subjects who were successfully extubated measured greater active distances $(p=0.014)$, and were able to cycle for longer $(p=0.013)$ than subjects who failed extubation (Table 3 ).

The MIP, MEP, RSBI and $\mathrm{PaO}_{2} / \mathrm{FiO}_{2}$ ratio were not associated with successful extubation. Eight subjects experienced pain prior to muscle strength and endurance testing, but none withdrew from the study.

\section{Discussion}

The aim of the pilot study was to determine if selected upper-limb muscle strength and endurance are potential predictors of successful extubation. These measures of muscle strength and endurance have never been evaluated previously as possible predictors of successful extubation.

Despite being ready for an SBT, the mean age of subjects who failed SBT and extubation was 61 years. This is in agreement with Thille et al., ${ }^{14}$ who reported that patients older than 65 years had a high risk (>25\%) of extubation failure. However, 2 of our subjects who failed extubation were only 30 years old. One subject was diagnosed with systemic lupus erythematosus and the other subject had undergone head and neck surgery. Systemic lupus erythematosus may cause physiological effects such as pulmonary hypertension, fatigue or myalgia, leading to failed extubation. The surgical patient suffered from upper airway obstruction post extubation. Graboyes et al..$^{[24]}$ found that $33 \%$ of patients who were intubated after head and neck surgery failed extubation owing to upper airway oedema. The inclusion of patients with these conditions will have to be reconsidered in further studies to determine predictors for extubation.

The results from the pilot study with 30 subjects indicate significant association between successful extubation and the muscle strength of 
Table 1. Subject demographics and diagnosis

\begin{tabular}{|c|c|c|}
\hline & Successful extubation $(n=23)$ & Failed SBT/failed extubation $(n=7)$ \\
\hline Male & $11(47.8 \%)^{*}$ & $1(14.3 \%)^{*}$ \\
\hline Female & $12(52.2 \%)$ & $6(85.7 \%)$ \\
\hline Age & $45.0(15.88)^{\dagger}$ & $61.0(20.07)^{\dagger}$ \\
\hline Days in ICU & $5.0(4.0 ; 8.0)^{\S}$ & $11.0(7.0 ; 15.5)^{\S}$ \\
\hline Days ventilated & $5.0(3.0 ; 7.0)$ & $8.0(4.5 ; 10.0)$ \\
\hline \multicolumn{3}{|l|}{ Diagnosis } \\
\hline Renal failure & $7(24 \%)^{*}$ & $1(3 \%)^{*}$ \\
\hline General and thoracic surgery & $4(17 \%)$ & $3(10 \%)$ \\
\hline Polytrauma & $2(7 \%)$ & $1(3 \%)$ \\
\hline Acute and chronic lung pathology & $3(10 \%)$ & $0(0 \%)$ \\
\hline Vascular surgery & $2(7 \%)$ & $0(0 \%)$ \\
\hline Organophosphate overdose & $2(7 \%)$ & $0(0 \%)$ \\
\hline Burns & $1(3 \%)$ & $0(0 \%)$ \\
\hline Systemic lupus erythematosus & $0(0 \%)$ & $1(3 \%)$ \\
\hline Below-knee amputation & $1(3 \%)$ & $0(0 \%)$ \\
\hline Commando surgery & $0(0 \%)$ & $1(3 \%)$ \\
\hline
\end{tabular}

Table 2. Muscle strength testing of trapezius, deltoid and neck flexor muscles

\begin{tabular}{|c|c|c|c|c|c|}
\hline Muscles tested & Successful extubation $(n=23)^{*}$ & Failed SBT $(n=5)^{*}$ & Failed extubation $(n=2)^{*}$ & $p$-value SBT & $\begin{array}{l}p \text {-value } \\
\text { failed } \\
\text { extubation }\end{array}$ \\
\hline \multicolumn{6}{|l|}{ Trapezius left } \\
\hline Grade 0 & 8.00 & 0.00 & 50.00 & \multirow{4}{*}{0.563} & \multirow{4}{*}{0.055} \\
\hline Grade 1 & 12.00 & 20.00 & 50.00 & & \\
\hline Grade 2 & 8.00 & 20.00 & 0.00 & & \\
\hline Grade 3 & 72.00 & 60.00 & 0.00 & & \\
\hline \multicolumn{6}{|l|}{ Trapezius right } \\
\hline Grade 0 & 8.00 & 0.00 & 50.00 & \multirow{4}{*}{0.728} & \multirow{4}{*}{0.067} \\
\hline Grade 1 & 12.00 & 20.00 & 50.00 & & \\
\hline Grade 2 & 12.00 & 20.00 & 0.00 & & \\
\hline Grade 3 & 68.00 & 60.00 & 0.00 & & \\
\hline \multicolumn{6}{|l|}{ Deltoid left } \\
\hline Grade 1 & 16.00 & 0.00 & 100.00 & \multirow{3}{*}{0.052} & \multirow{3}{*}{0.014} \\
\hline Grade 2 & 36.00 & 100.00 & 0.00 & & \\
\hline Grade 3 & 48.00 & 0.00 & 0.00 & & \\
\hline \multicolumn{6}{|l|}{ Deltoid right } \\
\hline Grade 0 & 4.00 & 0.00 & 50.00 & \multirow{4}{*}{0.106} & \multirow{4}{*}{0.011} \\
\hline Grade 1 & 12.00 & 40.00 & 50.00 & & \\
\hline Grade 2 & 36.00 & 60.00 & 0.00 & & \\
\hline Grade 3 & 48.00 & 0.00 & 0.00 & & \\
\hline \multicolumn{6}{|c|}{ Neck flexor muscle group } \\
\hline Grade 0 & 4.00 & 0.00 & 50.00 & \multirow{4}{*}{0.217} & \multirow{4}{*}{0.009} \\
\hline Grade 1 & 12.00 & 20.00 & 50.00 & & \\
\hline Grade 2 & 40.00 & 80.00 & 0.00 & & \\
\hline Grade 3 & 44.00 & 0.00 & 0.00 & & \\
\hline
\end{tabular}

the deltoid and neck flexor muscles. Muscle strength of the deltoid, neck flexors and trapezius muscles was tested with the Oxford grading scale by standardising of testing positions to ensure reliable muscle strength testing. This was done in agreement with Vanpee et al., ${ }^{[18]}$ who reported that the position of the patient, the limb and the angle of the joint should be included in the standardisation of the testing positions. Testing muscle strength with the Oxford grading scale in the present study represented results that were distributed over the range of muscle grades in the frequency columns. Comparison of measures (symmetry) was high (Kappa $=0.7931)$, indicating a high level of intra-rater reliability; this indicated that the Oxford grading scale can be considered as a tool to evaluate individual muscle strength in ICU. We believe that standardisation of the testing position and procedure helped to produce significant results. Currently, no literature is available regarding the assessment of muscle strength of deltoid, neck flexors and trapezius with the Oxford grading scale in ICU. Testing of the muscle strength of 


\begin{tabular}{llllll}
\hline & & & & & \multicolumn{1}{c}{$\begin{array}{c}p \text {-value SBT } \\
\text { and failed } \\
\text { extubation }\end{array}$} \\
\hline Variables tested & Total patients $(\boldsymbol{N}=\mathbf{3 0})$ & Successful extubation $(\boldsymbol{n}=23)$ & Failed SBT $(\boldsymbol{n}=5)$ & Failed extubation $(\boldsymbol{n}=2)$ & 0.014 \\
Passive distance $(\mathrm{km})$ & $0.29(0.0 ; 0.8)^{*}$ & $0.52(0.01 ; 0.86)^{*}$ & $0.0(0.0 ; 0.0)^{*}$ & $0.0(0.0 ; 0.0)^{*}$ & 0.014 \\
Active time (minutes) & $2.24(0.0 ; 0.48)$ & $0.09(0.0 ; 0.47)$ & $0.48(0.48 ; 0.49)$ & $0.48(0.47 ; 0.49)$ & 0.013 \\
Passive time (minutes) & $2.37(0.02 ; 4.57)$ & $4.12(0.13 ; 5.0)$ & $0.0(0.0 ; 0.09)$ & $0.0(0.0 ; 0.1)$ & 0.011 \\
$\mathrm{MIP}\left(\mathrm{cmH}_{2} \mathrm{O}\right)$ & $-23.0(-29.0 ;-17.0)$ & $-25.0(-35.0 ;-18.0)$ & $-17.0(-22.5 ;-14.0)$ & $-17.0(-19.0 ;-11.0)$ & 0.180 \\
$\mathrm{MEP}\left(\mathrm{cmH}_{2} \mathrm{O}\right)$ & $20.0(14.0 ; 25.0)$ & $20.0(16.0 ; 26.0)$ & $17.5(13.0 ; 22.0)$ & $19.5(14.0 ; 23.0)$ & 0.394 \\
$\mathrm{PaO}_{2} / \mathrm{FiO}_{2}$ ratio $(\mathrm{mmHg})$ & $254.0(207.0 ; 321.0)$ & $266.0(207.0 ; 329.0)$ & $234 .(218.0 ; 248.0)$ & $218.0(187.0 ; 248.0)$ & 0.388 \\
$\mathrm{RSBI}$ & $38.0(25.0 ; 65.0)$ & $37.0(25.0 ; 50.0)$ & $49.0(49.0 ; 86.0)$ & $49.0(35.0 ; 86.0)$ & 0.242
\end{tabular}

individual muscles such as the deltoid, neck flexors or trapezius with the Oxford grading scale was conducted taking into account the combined nerve innervations of the respiratory muscles and the deltoid, neck flexors and trapezius muscles via the corticospinal pathway and the possibility of interneuron activation $(\mathrm{C} 1-\mathrm{C} 6) .^{[12,13]}$ The authors are of the opinion that the MRC sum score should be added in future studies to have an indication of ICU-acquired weakness (ICU-AW) and the effect on the weaning and extubation of the subjects.

A possible explanation for the difference in muscle strength results between the deltoid, neck flexors and trapezius muscles may be due to the functional and physiological differences of the muscles. Functionally the neck flexor muscles are active during neck flexion, the deltoid during shoulder abduction, and the trapezius muscle is a cervical stabiliser. ${ }^{[25]}$ Physiologically, the neck flexors (specifically sternocleidomastoid) and deltoid are not affected by pain or dysfunction due to the predominant Type II muscle fibres. However, the trapezius has the tendency to atrophy in the presence of pain or dysfunction owing to Type I fibres. Electromyographic (EMG) activity of the trapezius muscle showed a nonlinear correlation and the sternocleidomastoid muscle showed a strong linear correlation with the MIP. ${ }^{[13]}$ Thus the nature of the relationship between the respiratory and the deltoid and neck flexor muscles may vary from that of the respiratory and trapezius muscles.

The deltoid, neck flexors, trapezius and respiratory muscles have combined nerve innervations via the corticospinal pathway. By strengthening the first-mentioned muscles, it was our hypothesis that there might be overflow of strength to the respiratory muscles. Evidence showed that interneuronal processing is involved in the organisation of motor outflow for respiration. ${ }^{[13]}$ The strength of the inspiratory and respiratory muscles may be enhanced by exercising muscles sharing the same nerve innervations, but concrete evidence requires a randomised controlled trial with a larger sample size. Bissett et al. ${ }^{[26]}$ demonstrated that respiratory muscle endurance is impaired owing to inspiratory muscle weakness following mechanical ventilation. Furthermore, Peñuelas et al. ${ }^{[27]}$ reported that a decrease in respiratory muscle strength determined the ability of patients to be successfully weaned from mechanical ventilation. The $\mathrm{RSBI}, \mathrm{PaO}_{2} / \mathrm{FiO}_{2}$ ratio, MIP and MEP measurements in our study did not demonstrate an association with successful extubation.

Endurance, measured using the MOTOmed letto2 cycle ergometer, also showed an association with successful extubation. Subjects in this study who actively rode a mean distance of $0.5 \mathrm{~km}$ in 4.12 minutes with the MOTOmed letto2 cycle ergometer had a significant association ( $p=0.014)$ with successful extubation. After 5 minutes, patients who used the cycle ergometer had small increases in HR, RR and sensation of dyspnoea. ${ }^{[28]}$ The patients also showed a high level of acceptance for using the MOTOmed letto2 cycle ergometer in ICU. ${ }^{[28]}$ The MOTOmed letto2 cycle ergometer has been used for rehabilitation purposes to improve the muscle strength and endurance of critically ill patients in ICU. It enhances muscle strength and increases the functional status and quality of life at hospital discharge. ${ }^{[28]}$ To the best of our knowledge, our study is the first to evaluate the use of the MOTOmed letto2 cycle ergometer as an assessment tool in mechanically ventilated patients. Hol et al ${ }^{[21]}$ tested the validity and reliability of the 6-minute arm test (6-MAT) in a group of patients with spinal injuries. They reported that the test had acceptable values for test-retest reliability and validity. ${ }^{[21]}$ Endurance testing on healthy persons can be used to determine an individual's ability to sustain high-intensity exercise for longer than 4 minutes. ${ }^{[20,21]}$ Due to the subjects being critically ill, the researchers tested each subject for only 5 minutes to reach steady state and not up to exhaustion on fatigue-untrained individuals.

Functional recovery in this study was aided by an early mobilisation protocol. Given the complex nature of the critical care setting, it is difficult to assign causality to extubation failure. This study showed possible associations of muscle strength and endurance with successful weaning.

\section{Study limitations}

The small sample of subjects may have contributed to the nonsignificance of the results of upper trapezius muscle strength testing. Muscle testing was not conducted in any specific order and should be standardised. The neck flexor muscle group should be separated to test individual muscles and not as a group. Subjects with prolonged mechanical ventilation were not formally assessed for ICU-AW, which could lead to extubation failure. The heart rate, saturation and blood pressure of subjects need to be documented before and after endurance testing to ensure standardisation of the testing procedure. MIP and MEP were measured with a limited number of ventilators, which limited sample size and significance. Testing muscle endurance before MIP and MEP measurements could contribute to inaccurate MIP and MEP readings owing to tiredness. We did not test for factors that may have confounded the relationship between muscle weakness and successful extubation. The total number of subjects excluded were not documented as this was a pilot study.

\section{Recommendations}

Future studies with larger sample sizes are needed to determine if muscle strength of the pectoralis major is associated with successful extubation. Larger sample sizes, with equal age distributions, are needed for comparisons between successfully extubated and failed extubated groups. 
The MRC score and hand-held dynamometry should be included in future studies to test ICU-AW and muscle strength. Future studies should test endurance after a set resting time following strength tests. Subjects should be familiarised with the MOTOmed letto2 cycle ergometer before commencing testing. Future studies should rather use standardised MIP measurement with electronical manometer than ventilators. Further research into the neurophysiological association between the respiratory muscles and the peripheral muscles is recommended. We suggest that the pain scale be used as an outcome after testing.

\section{Conclusion}

Accurate prediction of successful safe extubation is the ultimate goal in mechanically ventilated patients as it could influence the ICU and hospital length of stay and financial costs, and it could increase the patient's functional ability and quality of life post hospital discharge. The results from this pilot study with 30 subjects suggest an association between successful extubation and muscle strength of the deltoid and neck flexor muscles. The results also suggest an association between muscle endurance and successful extubation. Therefore, incorporating muscle strength and endurance testing into weaning protocols and physiotherapy rehabilitation programmes could lead to greater extubation success.

Acknowledgements. We thank the staff of the participating intensive care units and patients who made this study possible. None of these individuals received financial compensation for their contribution to this study. We also thank the two critical care clinical technologists (Ms C van Heerden and Ms M van Jaarsveld) for assisting with the MIP and MEP measurements. We thank Prof. K van der Meyden for his support and guidance in the study. Author contributions. CRdB: literature search, data collection, study design, analysis of data, manuscript preparation. AJvR: study design, analysis of data, review of manuscript. JPP: study design, review of manuscript. PJB, PR: data analysis. FP: review of manuscript. All authors agree to be accountable for all aspects of the work and have read and approved the manuscript.

\section{Funding. None.}

\section{Conflicts of interest. None}

1. Moodie LH, Reeve JC, Vermeulen N, Elkins MR. Inspiratory muscle training to facilitate weaning from mechanical ventilation: Protocol for a systematic review. BMC Res Notes 2011;4:283. https:// doi.org/10.1186/1756-0500-4-283

2. Diaz MC, Ospina-Tascón GA, Salazar BC. Respiratory muscle dysfunction: A multicausal entity in the critically ill patient undergoing mechanical ventilation. Arch Bronconeumol 2014;50(2):73-77. https://doi.org/10.1016/j.arbres.2013.03.005

3. Martin AD, Smith BK, Davenport PD, et al. Inspiratory muscle strength training improves weaning outcome in failure to wean patients: A randomized trial. Critical Care 2011;15(2):R84. https://doi. org/10.1186/cc10081.
4. Chang AT, Boots RJ, Brown MG, Paratz J, Hodges PW. Reduced inspiratory muscle endurance following successful weaning from prolonged mechanical ventilation. CHEST 2005;128(2):553559. https://doi.org/10.1378/chest.128.2.553

5. Seely AJE, Bravi A, Herry C, et al. Do heart and respiratory rate variability improve prediction of extubation outcomes in critically ill patients? Critical Care 2014;18(2):R65. https://doi.org/10.1186/ cc13822

6. Grosu HB, Lee YI, Lee J, Eden E, Eikermann M, Rose KM. Diaphragm muscle thinning in patients who are mechanically ventilated. CHEST 2012;142(6):1455-1460. https://doi.org/10.1378/ chest.11-1638

7. Thille AW, Richard JCM, Brochard L. The decision to extubate in the intensive care unit. Am J Respir Crit Care Med 2013;187(12):1294-1302. https://doi.org/10.1164/rccm.201208-1523CI

8. Thille AW, Cortés-Puch I, Esteban A. Weaning from the ventilator and extubation in ICU. Curr Opin Crit Care 2013;19:57-64. https://doi.org/10.1097/mcc.0b013e32835c5095

9. Frutos-Vivar F, Ferguson ND, Esteban A, et al. Risk factors for extubation failure in patients following a successful spontaneous breathing trial. CHEST 2006;130(6):1664-1671. https://doi. org/10.1378/chest.130.6.1664

10. De Jonghe B, Bastuji-Garin S, Durand MC, et al. Respiratory weakness is associated with limb weakness and delayed weaning in critical illness. Crit Care Med 2007;35(9):2007-2015. https://doi org/10.1097/01.CCM.0000281450.01881.d8

11. Condessa RL, Brauner JS, Saul AL, Baptista M, Silva ACT, Vieira SRR. Inspiratory muscle training did not accelerate weaning from mechanical ventilation but did improve tidal volume and maximal respiratory pressures: A randomised trial. J Physiotherapy 2013;59(2):101-107. https:/ doi.org/10.1016/S1836-9553(13)70162-0

12. Martini FH. Fundamentals of Anatomy \& Physiology. 6th ed. San Francisco: Benjamin Cummings; 2004: $358,361,430-433,440-444,848-849$

13. Terson de Paleville DGL, McKay WB, Folz RJ, Ovechkin AV. Respiratory motor control disrupted by spinal cord injury: mechanisms, evaluation and restoration. Transl Stroke Res 2011;2(4):463-473.

14. Thille AW, Boissier F, Ghezala HB, et al. Risk factors for and prediction by caregivers of extubation failure in ICU patients: A prospective study. Crit Care Med 2015;43(3):613-620. https://doi. org/10.1097/CCM.0000000000000748

15. Chlif M, Keochkerian D, Choquet D, Vaidie A, Ahmaidia S. Effects of obesity on breathing pattern, ventilator neural drive and mechanics. Respir Physiol Neurobiol 2009;168(3):198-202. https://doi. org/10.1016/..resp.2009.06.012

16. Wesley Ely E. Inouye SK, Bernard GR, et al. Delirium in mechanically ventilated patients: Validity and reliability of the Confusion Assessment Method for the Intensive care unit (CAM-ICU). JAMA 2001;286(21):2703-2710.

17. Van Aswegen H, Morrow B. Cardiopulmonary Physiotherapy in Trauma: An Evidence-based Approach. London: Imperial College Press; 2015:68-72.

18. Vanpee G, Hermans G, Segers J, Gosselink R. Assessment of limb muscle strength in critically ill patients: A systematic review. Crit Care Med 2014;42(3):701-711. https://doi.org/10.1097/ CCM.0000000000000030

19. Kendall FP, McCreary EK, Provance PG, McIntyre Rodgers M, Romani WA. Muscle testing and function with Posture and Pain. 5th ed. Philadelphia: Lippincott Williams \& Wilkins; 2005.

20. McArdle WD, Katch FI, Katch VL. Exercise Physiology. 4th ed. Philadelphia: Williams \& Wilkins; 1996.

21. Hol AT, Eng JJ, Miller WC, et al. Reliability and validity of the 6-minute arm test for the evaluation of cardiovascular fitness in individuals with spinal cord injury. Arch Phys Med Rehabil 2007;88(4):489-495. https://doi.org/10.1016/j.apmr.2006.12.044

22. StataCorp. 2015. Stata Statistical Software: Release 13. College Station: StataCorp.

23. R 3.1.1 on Windows. Internet. Available from www.r-statistics.com

24. Graboyes EM, Bradley JP, Kallogjeri D, Cavallone LF, Nussenbaum B. Prognosis and patterns of failure for the extubation of patients who remain intubated after head and neck surgery. Ann Otol Rhinol Laryngol 2015;124(3):179-186. https://doi.org/10.1177/0003489414549576

25. Levangie PK, Norkin CC. Joint Structure and Function: A Comprehensive Analysis. 3rd ed. Philadelphia: F.A. Davis Company; 2001:85-93.

26. Bissett B, Leditschke IA, Neeman T, Boots R, Paratz J. Weaned but weary: One third of adult intensive care patients mechanically ventilated for 7 days or more have impaired inspiratory muscle endurance after successful weaning. Heart Lung 2015;44(1):15-20. http://doi.org/10.1016/j. hrtlng.2014.10.001

27. Peñuelas Ó, Thille AW, Esteban A. Discontinuation of ventilator support: New solutions to old dilemmas. Curr Opin Crit Care 2015;21(1):74-81. https://doi.org/10.1097/mmc.0000000000000169

28. Pires-Neto RC, Pereira AL, Parente C, et al. Characterization of the use of a cycle ergometer to assist in the physical therapy treatment of critically ill patients. Rev Bras Ter Intensiva 2013;25(1):39-43.

Accepted 19 October 2018 